\title{
Automated Mineralogy and Quantitative Compositional Analysis of Geological Samples on a Multi-Functional Scanning Electron Microscope
}

\author{
Rosie Jones, Matt Hiscock, Hui Jiang and Alexandra Stavropoulou \\ Oxford Instruments, High Wycombe, England, United Kingdom
}

The scanning electron microscope (SEM) is a common and powerful tool, which, when combined with an analytical system, can perform complete characterisations of geological materials in a single analytical session. In this study we demonstrate how a standard, multi-functional SEM equipped with an energy dispersive x-ray spectrometry (EDS) system can be used to automatically determine mineral elemental compositions and textural relationships, in a fast and effective manner. We present results from a range of geological samples, demonstrating flexible whole sample (polished blocks, thin sections, grain mounts) characterisation, as well as rapid identification and location of key minerals of interest (e.g., zircon, monazite) for further targeted analyses both inside (e.g., quantitative EDS) and outside of the SEM (e.g., radiometric dating). We also demonstrate the advantage of using a standard SEM platform for automated mineralogy, as the results can be quickly correlated with other imaging or analytical techniques, such as electron backscatter diffraction (EBSD).

Analysis of geological samples on a multi-functional SEM has the major advantage over other techniques commonly used to determine mineral abundance and chemical composition (e.g., XRD, XRF, ICP-MS) of being non-destructive, meaning samples can be re-analysed/analysed using other in-situ techniques (e.g., SIMS). In-situ and automated mineral analysis on an SEM allows workers investigating rock samples (e.g., in the mineral exploration/processing industry or geological research) to obtain information on; grain size and morphology; texture (e.g., mineral relationships and associations); mineral chemical composition and variability; relative mineral abundance; and porosity. In addition, automated mineral analysis, combined with a mineral classification scheme can replace traditional optical point counting, which is laborious and prone to bias.

The samples analysed include thin sections of igneous rocks from the southern Central Andes, and mineral grain mounts typical of the type used in the mining industry. These samples were analysed on a Tescan S8000 FEG-SEM (at 20kV accelerating voltage) combined with an Oxford Instruments Ultim Max $170 \mathrm{~mm}^{2}$ EDS detector and AZtec software (including AZtecMineral for automated mineralogy). The following guided workflow is used for automated mineral analysis:

1. A back-scatter electron (BSE) image is captured for a representative area of the sample with the SEM contrast/brightness optimised to give the maximum spread in greyscale for the minerals of interest (i.e., low average atomic number minerals are close to black, and high average atomic number minerals are white).

2. The sample matrix and/or pore space (i.e., black in the BSE image) is defined and removed and particle separation parameters/filters are set.

3. Thresholds are set up, based on the BSE image greyscale, to identify and separate the different mineral types present in the sample.

4. Particle detection and EDS acquisition is run over this small sample area and the results checked for suitability.

5. The minerals for which EDS data is acquired are then classified using an editable, mineral classification scheme.

6. An automated run is set up to detect particles and acquire EDS data from a large sample area (e.g., an entire thin section).

7. The mineral dataset is exported and analysed in post-processing software (GrainAlyser2). 
An example result is shown in Figure 1.

We go on to optimise the EDS system for quantitative, 'standardless' EDS analysis. This technique determines quantitative mineral compositions based on inbuilt standards sets, which contain experimental $\mathrm{X}$-ray intensity ratios for numerous, well-known standard materials. This data was collected as part of the development of the EDS system by experts using a strict methodology and a well characterised EDS detector. It negates the need for the end-user to obtain, maintain, and analyse a wide range of standards in order to obtain quantitative, un-normalised results. In order to perform such analysis, the user simply needs to collect a beam current measurement on a single, un-oxidised, pure element such as copper (assuming that the sample to be analysed is flat, polished, coated in a conductive layer, and at the optimal microscope working distance). We compare these un-normalised EDS results with results obtained on the same mineral grains and samples by electron microprobe (EPMA) and show that a comparable level of accuracy and reproducibility can be achieved.

These quantitative EDS results for amphibole and plagioclase are then applied to a range of amphiboleplagioclase geothermobarometers [e.g., 1,2] to determine the pressure and temperature conditions at which igneous rocks from the southern Central Andes equilibrated and crystallised. This information is also combined with textural information obtained from large area electron imaging and EDS mapping, demonstrating the versatility of a multifunctional SEM combined with a high-performance EDS detector and the range of geological information that can be obtained.
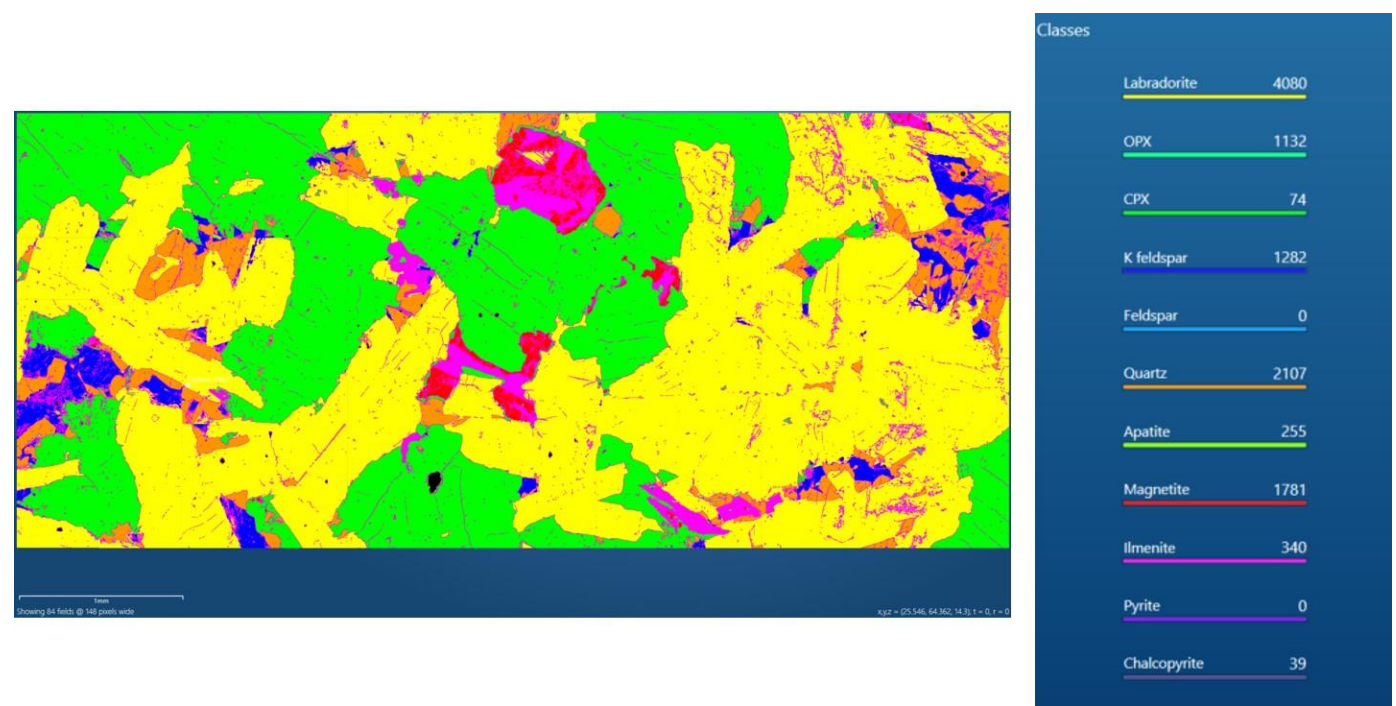

Figure 1. The mineral classification results of an automatically acquired large area run on an intrusive igneous rock sample, qualitatively showing mineral abundance and textural information, on which further sample analysis/processing can be based.

\section{References}

[1] Mutch, E. J. F., et al. "An experimental study of amphibole stability in low-pressure granitic magmas and a revised Al-in-hornblende geobarometer." Contributions to Mineralogy and Petrology 171.10 (2016): 85.

[2] Ridolfi, F., and Renzulli, A. "Calcic amphiboles in calc-alkaline and alkaline magmas: thermobarometric and chemometric empirical equations valid up to $1,130^{\circ} \mathrm{C}$ and $2.2 \mathrm{GPa}$." Contributions to Mineralogy and Petrology 163.5 (2012): 877-895. 